\title{
Pacific
}

Journal of

Mathematics

\section{PLANCHEREL FORMULAE FOR NON-SYMMETRIC POLAR}

\section{HOMOGENEOUS SPACES}

\author{
JING-SONG HUANG
}




\title{
PLANCHEREL FORMULAE FOR NON-SYMMETRIC POLAR HOMOGENEOUS SPACES
}

\author{
JING-SONG HUANG
}

Let $G$ be a semisimple Lie group and let $H$ be a closed reductive subgroup of $G$. The homogeneous space $X=G / H$ is called a semisimple homogeneous space. A fundamental goal of harmonic analysis is to understand the group action of $G$ on the various function spaces of $X$. In particular, the $L^{2}$-harmonic analysis on $X$ is to decompose $L^{2}(X)$ as a direct integral of irreducibles, i.e., to find a family of irreducible unitary representations $\left\{V_{\omega} \mid \omega \in \Omega\right\}$ of $G$, and a measure $\nu$ on the set $\Omega$, so that

$$
L^{2}(X) \cong \int_{\Omega} V_{\Omega} d \nu(\omega) \quad \text { (unitary } G \text {-isomophism) }
$$

The above decomposition is called the Plancherel formula for the homogeneous space $X$. In this paper we prove the Plancherel formulae for some non-symmetric semisimple homogenous spaces.

\section{Introduction.}

The equivalence classes of the representations of $G$ on the minimal closed invariant subspace of $L^{2}(X)$ is called the discrete series for $X$. The discrete series may not exist for some homogeneous spaces. In the case $X=G /\{e\}$ is a semisimple Lie group, Harish-Chandra proved that $G$ has discrete series if and only if the real rank of $G$ is equal to the rank of $K([\mathbf{H C}])$. In other words, $G$ has discrete series if and only if $G$ has a compact Cartan subgroup. HarishChandra also obtained a classification of the discrete series for semisimple Lie groups. In the case $X=G / H$ is a semisimple symmetric space, FlenstedJensen ([FJ]) proved that $G / H$ has discrete series if

$$
\text { rank of } G / H=\operatorname{rank} \text { of } K / K \cap H \text {. }
$$

Later on Oshima and Matsuki $([\mathbf{O M}])$ proved if $G / H$ has discrete series then it must satisfy the rank condition (1.2). That is to say, the semisimple symmetric space $G / H$ has discrete series if and only if it has a compact 
Cartan subspace. Oshima and Matsuki also gave a description of all discrete series for semisimple symmetric spaces. Since the group $G$ can be regarded as symmetric space $G \times G / \mathrm{d}(G)$, the results of Flensted-Jensen, Othima and Matsuki are the generalizations of that of Harish-Chandra.

It is then a natural question to ask when a semisimple homogeneous space $G / H$ has discrete series? Based on the observation of the results mentioned above, we would guess $G / H$ has discrete series if and and if it has a compact Cartan subspace. Then we face the problem how to define a Cartan subspace for a semisimple homogeneous space. The author succeeded in definiting Cartan subspaces for a class of semisimple homogeneous spaces in $[\mathbf{H}]$. These spaces are called polar spaces and they are natural generalizations of semisimple symmetric spaces. In this paper we prove that a polar $G / H$ has discrete series if and only if it has a compact Cartan subspace. The main purpose of the paper is to describe all discrete series and prove the Plancherel formulae for non-symmetric polar spaces.

The method we use is as follows. As we will recall in Section 2 the simple non-symmetric noncompact polar spaces are essentially $\operatorname{Spin}_{e}(3,4) / \mathrm{G}_{2(2)}$, $\mathrm{G}_{2(2)} / \mathrm{SU}(2,1)$ and $\mathrm{G}_{2(2)} / \mathrm{SL}(3, \mathbb{R})$. These non-symmetric polar homogeneous space are all isomorphic to some real hyperbolic symmetric spaces $G^{\prime} / H^{\prime}=\mathrm{SO}_{e}(p, q) / \mathrm{SO}_{e}(p, q-1)$. We have

$$
\begin{aligned}
\operatorname{Spin}_{e}(3,4) / \mathrm{G}_{2(2)} & \cong \mathrm{SO}_{e}(4,4) / \mathrm{SO}_{e}(4,3), \\
\mathrm{G}_{2(2)} / \mathrm{SU}(2,1) & \cong \mathrm{SO}_{e}(4,3) / \mathrm{SO}_{e}(4,2), \\
\mathrm{G}_{2(2)} / \mathrm{SL}(3, \mathbb{R}) & \cong \mathrm{SO}_{e}(3,4) / \mathrm{SO}_{e}(3,3) .
\end{aligned}
$$

Moreover, these isomorphisms are induced by the embedding of $G$ into $G^{\prime}$, if we denote by $G^{\prime} / H^{\prime}$ the corresponding real hyperbolic spaces. It is our strategy to make use of the powerful results $([\mathbf{R}])$ on real hyperbolic spaces to obtain the decomposition (1.1) for polar spaces. The magic is that any irreducible unitary representation of $G^{\prime}$ occurring in the decomposition for real hyperbolic space remains irreducible when it is restricted to $G$, no matter if it is in the continuous spectrum or in the set of discrete series. Therefore the decomposition (1.1) for some real hyperbolic spaces induces the decomposition for polar spaces. The strategy used in this paper may be generalized to study various harmonic analysis problems, such as discrete series, on a larger class of semisimple homogeneous spaces by using known results on semisimple symmetric spaces.

E. Heintze, R. Palais, C.-L. Terng and G. Thorbergsson have done an extensive study of isometric actions on polar Riemannian manifold and $k$-flat homogeneous spaces [HPPT]. T. Kobayashi and T. Uzawa $[\mathbf{K}]$ obtained a description of discrete series of $\mathrm{G}_{2(2)} / \mathrm{SU}(2,1)$ and $\mathrm{G}_{2(2)} / \mathrm{SL}(3, \mathbb{R})$ in a 
different parameters by their beautiful method of discrete decomposition of the restriction of $A_{\mathfrak{q}}(\lambda)$-modules to reductive subgroups. Kobayashi [K] also proved the existence of discrete series for a lot of non-symmetric homogeneous spaces.

This paper is organized as following. In Section 2 we recall the definition of polar spaces. In Section 3 we define the principal series for $X$. In Section 4 we obtain Plancherel formula for $X$. In Section 5 we discuss the representations of eigenspace spaces of $G$-invariant differential operators on $X$ and obtain a Poisson integral formula for $K$-finite eigenfunctions. The author would like to thank Nolan Wallach for helpful discussions.

\section{Polar spaces and real hyperbolic spaces.}

Suppose $G$ is a connected semisimple compact Lie group and $H$ a closed subgroup. In order for the homogeneous space $G / H$ to have the same kind of properties of a compact symmetric space, the first thing we expect is that $G / H$ has a Cartan subspace. Let $\mathfrak{g}$ and $\mathfrak{h}$ denote the Lie algebras of $G$ and $H$ respectively and $\mathfrak{q}$ the orthogonal complement of $\mathfrak{h}$ in $\mathfrak{g}$ with respect to the Killing form of $\mathfrak{g}$. The tangent space of $G / H$ at the identity $e H$ can be identified with the vector space $\mathfrak{q}$. We want that all maximal abelian subalgebras contained in $\mathfrak{q}$ are conjugated by the action of $H$. This action of $H$ is the adjoint action of $G$ restricted to $H$ and will be denoted by $\operatorname{Ad}_{G}(H)$. Note that the representation space of $\operatorname{Ad}_{G}(H)$ is $\mathfrak{q}$, which is a vector space over $\mathbb{R}$. It is very natural to require $\operatorname{Ad}_{G}(H)$ on $\mathfrak{q}$ to be a polar representation as defined below (Definition 2.2).

Let $H$ be a compact Lie group and $\mathfrak{h}$ be its Lie algebra. Consider a representation of $H$ on a real vector space $V$. Let $\langle$,$\rangle be a H$-invariant inner product on $V$. For a vector $v \in V$ let $a_{v}$ be the subspace of $V$ defined by

$$
a_{v}=\{w \in V \mid\langle w, \mathfrak{h} \cdot v\rangle=0\} .
$$

In other words, $a_{v}$ is the normal space to the $H$-orbit $H \cdot v$ at $v$. A easy fact about $a_{v}$ is that it meets every $H$-orbit. A vector $v \in V$ is called regular if $\mathfrak{h} \cdot v$ is of maximal possible dimension.

Proposition 2.1 [D]. Fix a regular vector $v_{0} \in V$. The following conditions are equivalent:

(i) For any regular vector $v \in V, \mathfrak{h} \cdot v=h \cdot\left(\mathfrak{h} \cdot v_{0}\right)$ for some $h \in H$.

(ii) For any regular vector $v \in V, a_{v}=h \cdot a_{v_{0}}$ for some $h \in H$.

(iii) For any $w \in a_{v_{0}},\left\langle\mathfrak{h} \cdot w, a_{v_{0}}\right\rangle=0$.

Definition 2.2. A representation of $H$ on $V$ is called polar if it satisfies one of the three equivalent conditions in Proposition 2.1. If $v \in V$ is regular then 
the subspace $a_{v}$ is called a Cartan subspace. Let $a$ be a Cartan subspace. The Weyl group $W$ of the polar representation $(\pi, V)$ is defined as $N_{H}(a) / Z_{H}(a)$.

Here are a few examples of polar representations:

(a) The natural representations of the classical compact Lie groups.

(b) The nontrivial representations of the exceptional compact Lie groups of minimal possible dimension.

(c) The adjoint representations.

(d) The action of $\operatorname{Ad}_{G}(H)$ on $\mathfrak{g} / \mathfrak{h}$ provided $G / H$ is a symmetric space.

Definition 2.3. Let $G$ be a semisimple compact Lie group with Lie algebra $\mathfrak{g}$ and $H$ a closed subgroup of $G$ with Lie algebra $\mathfrak{h}$. We say $(\mathfrak{g}, \mathfrak{h})$ is a simple polar pair if the action of $\operatorname{Ad}_{G}(H)$ on $\mathfrak{g} / \mathfrak{h}$ is an irreducible polar representation. In general, a Lie algebra pair $(\mathfrak{g}, \mathfrak{h})$ is call polar, if $\mathfrak{g}=$ $\oplus_{i} \mathfrak{g}_{i}, \mathfrak{h}=\oplus_{i} \mathfrak{h}_{i}$ such that $\left(\mathfrak{g}_{i}, \mathfrak{h}_{i}\right)$ is a polar pair for all $i$ 's. The corresponding homogeneous space $G / H$ is called a polar space.

From representation-theoretic point of view the polar spaces are more natural subjects to study than the symmetric spaces. The Cartan subspace $\mathfrak{a}$ and Weyl group $W$ of a polar space can be defined by the corresponding notions of polar representations. All the important results for compact symmetric spaces can be extended to compact polar spaces. For instance we can show that there exists an algebra isomorphism from the set of invariant differential operators on a polar space onto the $W$-invariant elements $S\left(\mathfrak{a}_{\mathbb{C}}\right)^{W}$ of symmetric algebra $S\left(\mathfrak{a}_{\mathbb{C}}\right)$ over $\mathfrak{a}_{\mathbb{C}}$. This result is even true for noncompact polar spaces since it does not depends on the various real forms. If $(G, H)$ is a compact polar pair with $G$ simply connected and $H$ connected, we can describe the irreducible representations of $G$ with a $H$-fixed vectors in the same way as for symmetric pairs.

It is clear that all compact simple symmetric pairs are compact simple polar pairs. Therefore all compact symmetric spaces are compact polar spaces. Besides symmetric pairs there are only two simple polar pairs $(\mathfrak{g}, \mathfrak{h})$ of Lie algebras up to automorphisms of $\mathfrak{g}$. They are $\left(B_{3}, \mathfrak{g}_{2}\right)$ and $\left(\mathfrak{g}_{2}, A_{2}\right)$ (cf. [H] Theorem 3.5.). Let $G$ be a linear semisimple group and $H$ be a closed reductive group. The homogeneous space $G / H$ is called a (non-compact) polar space if its compact real form $G^{c} / H^{c}$ is a compact polar space. Here $G^{c}$ and $H^{c}$ are compact Lie groups such that their complexifications are isomorphic to the complexifications of $G$ and $H$ respectively.

Theorem 2.4 ([H, Theorem 7.3]). Let $U / K$ be a simple non-symmetric compact polar space, with $U$ simply connected and both $G$ and $K$ connected. In other words, we assume that $U / K$ is either $G_{2(-14)} / S U(3)$ or 
$\operatorname{Spin}(7) / G_{2(-14)}$. Here $G_{2(-14)}$ is the simply connected compact Lie group with Lie algebra $\mathfrak{g}_{2}$. Then there are essentially three noncompact real forms $G / H$ of $U / K$ (which means $G_{\mathbb{C}}=U_{\mathbb{C}}$ and $K_{\mathbb{C}}=H_{\mathbb{C}}$ ). They are $G_{2(2)} / S U(2,1)$, $G_{2(2)} / S L(3, \mathbb{R})$ and $\operatorname{Spin}(3,4) / G_{2(2)}$. Here $G_{2(2)}$ is the noncompact real form of the complex group $G_{2}$.

In this paper we will prove the following theorem.

Theorem 2.5. Let $U / K$ be a compact polar space with $U$ connected and simple connected and $K$ connected. Let $G / H$ be a noncompact real form of $U / K$. Then $G / H$ has discrete series if and only if it has a compact Cartan subspace.

By Theorem 2.4 in order to prove the theorem it suffices to verify it for the three non-symmetric simple polar spaces $\mathrm{G}_{2(2)} / \mathrm{SU}(2,1), \mathrm{G}_{2(2)} / \mathrm{SL}(3, \mathbb{R})$ and $\operatorname{Spin}_{e}(3,4) / \mathrm{G}_{2(2)}$. Since all of the three polar spaces have compact Cartan subspaces, we will show that they all have discrete series. We actually obtain the Plancherel formulae for these homogeneous spaces in Section 4 . In particular, we obtain all discrete series for these homogeneous spaces.

The three non-symmetric polar spaces $X=G / H, \mathrm{G}_{2(2)} / \mathrm{SU}(2,1)$, $\mathrm{G}_{2(2)} / \mathrm{SL}(3, \mathbb{R})$ and $\operatorname{Spin}_{e}(3,4) / \mathrm{G}_{2(2)}$, are isomorphic to real hyperbolic spaces in a special way. Now we describe the isomorphisms. Consider the following three quadruplets:

1. Let $G=\operatorname{Spin}_{e}(3,4)$ be the subgroup of $G^{\prime}=\mathrm{SO}_{e}(4,4)$. Let $H^{\prime}=\mathrm{SO}_{e}(4,3)$ be the standard subgroup of $G^{\prime}$. Then $H=G \cap H^{\prime}$ is isomorphic to $\mathrm{G}_{2(2)}$.

2. Let $G=\mathrm{G}_{2(2)}$ be the subgroup of $G^{\prime}=\mathrm{SO}_{e}(4,3)$. Let $H^{\prime}=\mathrm{SO}_{e}(4,2)$ be the standard subgroup of $G^{\prime}$. Then $H=G \cap H^{\prime}$ is isomorphic to $\mathrm{SU}(2,1)$.

3. Let $G=\mathrm{G}_{2(2)}$ be the subgroup of $G^{\prime}=\mathrm{SO}_{e}(3,4)$. Let $H^{\prime}=\mathrm{SO}_{e}(3,3)$ be the standard subgroup of $G^{\prime}$. Then $H=G \cap H^{\prime}$ is isomorphic to $\operatorname{SL}(3, \mathbb{R})$.

\section{List of the Three Quadruplets.}

\begin{tabular}{|c|c|c|c|c|}
\hline & $\mathrm{G}$ & $\mathrm{G}^{\prime}$ & $\mathrm{H}^{\prime}$ & $\mathrm{H}$ \\
\hline 1. & $\mathrm{Spin}_{e}(3,4)$ & $\mathrm{SO}_{e}(4,4)$ & $\mathrm{SO}_{e}(4,3)$ & $\mathrm{G}_{2(2)}$ \\
\hline 2. & $\mathrm{G}_{2(2)}$ & $\mathrm{SO}_{e}(4,3)$ & $\mathrm{SO}_{e}(4,2)$ & $\mathrm{SU}(2,1)$ \\
\hline 3. & $\mathrm{G}_{2(2)}$ & $\mathrm{SO}_{e}(3,4)$ & $\mathrm{SO}_{e}(3,3)$ & $\mathrm{SL}(3, \mathbb{R})$ \\
\hline
\end{tabular}

In all of the three cases the inclusion of $G \hookrightarrow G^{\prime}$ induces a diffeomorphism of $G / H$ onto $G^{\prime} / H^{\prime}$. In other words, the natural embedding $G / H \hookrightarrow G^{\prime} / H^{\prime}$ is surjective. The very significance of these diffeomorphisms is that we can make use the known results on real hyperbolic spaces to obtain the desired results on polar spaces. 
For $q$ larger than 1 the homogeneous space $G^{\prime} / H^{\prime}=\mathrm{SO}_{e}(p, q) / \mathrm{SO}_{e}(p, q-1)$ may be realized as the hypersurfaces

$$
X=\left\{x \in \mathbb{R}^{p+q} \mid-x_{1}^{2}-\cdots-x_{p}^{2}+x_{p+1}^{2}+\cdots+x_{p+q}^{2}=1\right\}
$$

under the left action of the group $G^{\prime}=\mathrm{SO}_{e}(p, q)$ of connected linear transformations of $\mathbb{R}^{p+q}$ which leave the bilinear form $(x, y)=-x_{1} y_{1}-\cdots-$ $x_{p} y_{p}+x_{p+1} y_{p+1}+\cdots+x_{p+q} y_{p+q}$ invariant. Therefore the three polar spaces can be identified with $X$ for some proper integers $p$ and $q$ by restricting the action of $G^{\prime}$ to $G$. Follow the idea in $[\mathbf{R}]$ we introduce the asymptotic cone $\Xi=\left\{\xi \in \mathbb{R}^{p+q} \mid(\xi, \xi)=0, \xi \neq 0\right\}$ of $X$. Denote by $K$ and $K^{\prime}$ the maximal compact subgroup of $G$ and $G^{\prime}$ respectively. The manifold $Y=\left\{y \in \mathbb{R}^{p+q} \mid y_{1}^{2}+\cdots+y_{p}^{2}=y_{p+1}^{2}+\cdots+y_{p+q}^{2}=1\right\} \cong S^{p-1} \times S^{q-1} \subset \Xi$ may be regarded as a compact subspaces of the polar space, since $Y \cong$ $K / K \cap H \cong K^{\prime} / K^{\prime} \cap H^{\prime}$. Note that $Y$ is contained in the asymptotic cone $\Xi$, but not in $X$. The three cases we are interested have $p$ and $q$ equal either 3 or 4 . So in the rest of the paper, we always have both $p$ and $q$ larger than 1 .

\section{Principal series for $X$.}

We retain the notations in the previous section. Note that both $G^{\prime}$ and $G$ act transitively on $\Xi$, and the actions commute with the multiplicative action of nonzero real scalars $\mathbb{R}^{\times}$. Hence the space of functions on $\Xi$ which transform according to a fixed character of $\mathbb{R}^{\times}$is invariant under the regular representation of $G$ or $G^{\prime}$. So, for $\varepsilon=0,1$ and $\nu \in \mathbb{C}$, define

$$
\begin{gathered}
L_{\varepsilon, \nu}^{2}(\Xi)=\left\{f:\left.\Xi \rightarrow \mathbb{C}|f(\alpha \xi)=| \alpha\right|^{-\rho+\nu} \operatorname{sign}^{\varepsilon}(\alpha) f(\xi), \forall \alpha \in \mathbb{R}^{\times} ;\right. \\
\text {and } \left.\int_{Y}|f(y)|^{2} d y<\infty\right\},
\end{gathered}
$$

where $\rho=\frac{1}{2}(p+q-2)$. The representation $\left(\pi_{\varepsilon, \nu}, L_{\varepsilon, \nu}^{2}(\Xi)\right)$ is called a principal series for $X$.

\section{Proposition 3.1.}

(i) The pairing of $L_{\varepsilon, \nu}^{2}(\Xi)$ and $L_{\varepsilon,-\nu}^{2}(\Xi)$ defined by

$$
\left\langle\phi_{+}, \phi_{-}\right\rangle=\int_{Y} \phi_{+}(y) \phi_{-}(y) d y
$$

is $G^{\prime}$-invariant (and hence $G$-invariant).

(ii) The representation $\left(\pi_{\varepsilon, \nu}, L_{\varepsilon, \nu}^{2}(\Xi)\right)$ and $\left(\pi_{\varepsilon, \nu}, L_{\varepsilon, \nu}^{2}(\Xi)\right)$ are contragredient (as either representation of $G$ or $G^{\prime}$ ). 
(iii) The representation $\left(\pi_{\varepsilon, \nu}, L_{\varepsilon, \nu}^{2}(\Xi)\right)$ is unitary for imaginary $\nu$.

Proof. The statement (i) is proved in Lemma 1 in $[\mathbf{R}]$. The statement (ii) and (iii) are consequences of (i).

To study the irreducibility of $L_{\varepsilon, \nu}^{2}(\Xi)$ as a representation of $G$, we pass to its $K$-finite part which is stable under the action of $\mathfrak{g}$ obtained from $\pi_{\varepsilon, \nu}$ by derivation. First of all, the $K^{\prime}$-finite functions in $L_{\varepsilon, \nu}^{2}(\Xi)$ is isomorphic to $L_{\varepsilon}^{2}(Y)=\left\{\phi \in L^{2}(Y) \mid \phi(-y)=(-1)^{\varepsilon} \phi(y)\right\}$. Hence, as a representation of $K^{\prime}$

$$
L_{\varepsilon, \nu}^{2}(\Xi) \cong \sum_{\substack{m+n \equiv \varepsilon \\(\bmod 2)}} H^{m, n} \text { (Hilbert space sum) }
$$

where $H^{m, n}$ is the space spanned by the products of spherical harmonics of degree $m$ on $S^{p-1}$ with those of degree $n$ on $S^{p-1}$. As is well known, the $H^{m, n}$ 's are irreducible, pairwise inequivalent representations of $K^{\prime}$. These spherical harmonics are also irreducible representations of $K$ when the action of $K^{\prime}$ is restricted to $K$. We can prove this by using the case by case argument.

Theorem 3.2. A subspace of $\sum_{m+n \equiv \varepsilon \bmod 2} H^{m, n}$ is an irreducible $(\mathfrak{g}, K)$ module if and only if it is an irreducible $\left(\mathfrak{g}^{\prime}, K^{\prime}\right)$-module.

Proof. Since $\mathfrak{k}$ acts irreducibly on $\mathfrak{g} / \mathfrak{k}$, a subspace of $\sum_{m+n \equiv \varepsilon \bmod 2} H^{m, n}$ is $\mathfrak{g}$-stable if and only if it is stable under $\mathfrak{k}$ and a single element $X$ in $\mathfrak{g}$ not in $\mathfrak{k}$. The element $X \in \mathfrak{g}$ is automatically contained in $\mathfrak{g}^{\prime}$. The theorem follows from Theorem 2 of $[\mathbf{R}]$ regarding $L_{\varepsilon, \nu}^{2}(\Xi)$ as a $\left(\mathfrak{g}^{\prime}, K^{\prime}\right)$-module.

\section{Corollary 3.3.}

(i) The representation $\left(\pi_{\varepsilon, \nu}, L_{\varepsilon, \nu}^{2}(\Xi)\right)$ is an irreducible representation of $G$ or $G^{\prime}$ unless $-\rho+\nu$ is an integer.

(ii) If $-\rho+\nu$ is an integer, then for any pair $(u, v), u, v= \pm 1$, satisfying

$$
-\rho+\nu \equiv \varepsilon+\frac{1}{2}(u-1) p+\frac{1}{2}(v-1) q(\bmod 2)
$$

the closure of

$\Sigma\left\{H^{m, n} \mid u\left(m+\frac{1}{2}(p-2)\right)+v\left(n+\frac{1}{2}(q+2)\right)<\nu, m+n \equiv \varepsilon(\bmod \overline{2})\right\}$

is a $G$-stable subspace of $L_{\varepsilon, \nu}^{2}(\Xi)$, and any $G$-stable subspace is an intersection of some of these. 


\section{Plancherel formula for $X$.}

Now we introduce the polar coordinate on the polar space $X=G / H$ :

$$
(1, \infty) \times Y \rightarrow X,(\alpha, y) \mapsto x(\alpha, y)
$$

where

$$
x(\alpha, y)=\frac{1}{2}\left(\alpha-\alpha^{-1} y^{\prime}\right)+\frac{1}{2}\left(\alpha-\alpha^{-1} y^{\prime \prime}\right),
$$

if we decompose $y=y^{\prime}+y^{\prime \prime}$, with $y^{\prime} \in S^{p-1} \subset Y$ and $y^{\prime \prime} \in S^{q-1} \subset Y$ in the obvious way. This map is analytically invertible on its image $X^{\prime}=$ $\left\{x \in X \mid x_{1}^{2}+\cdots x_{p}^{2} \neq 0\right\}$.

The $G$-invariant differential operators on $X$ were described in $[\mathbf{H}]$. They are polynomials in $\Delta_{X}$, the Laplace operator on $X$. In polar coordinates, one has

$$
\begin{aligned}
\Delta_{X}= & \left(\alpha \frac{d}{d \alpha}\right)^{2}+\left((p-1) \frac{\alpha+\alpha^{-1}}{\alpha-\alpha^{-1}}+(q-1) \frac{\alpha-\alpha^{-1}}{\alpha+\alpha^{-1}}\right) \alpha \frac{d}{d \alpha} \\
& +\left(\frac{1}{2}\left(\alpha-\alpha^{-1}\right)\right)^{-2} \Delta_{S^{p-1}}-\left(\frac{1}{2}\left(\alpha+\alpha^{-1}\right)\right)^{-2} \Delta_{S^{q-1}}
\end{aligned}
$$

where $\Delta_{S^{p-1}}$ and $\Delta_{S^{q-1}}$ are the Laplacians on $S^{p-1}$ and on $S^{q-1}$ (cf. (4) in $[\mathbf{R}])$.

Denote by $D(X)$ the space of compactly supported smooth functions on $X$. The dual space $D^{\prime}(X)$ of $D(X)$ is the space of distributions on $X$. The Laplacian $\Delta_{X}$, considered as an operator on $D^{\prime}(X)$, can be restricted to a self-adjoint operators on $L^{2}(X)$ in a natural way.

Lemma 4.1 ([R] Lemma 9).

The Laplacian $\Delta_{X}$ is a self-adjoint operator on $L^{2}(X)$ with domain

$$
\operatorname{Dom}\left(\Delta_{X}\right)=\left\{f \in D^{\prime}(X) \mid f \in L^{2}(X) \text { and } \Delta_{X} f \in L^{2}(X)\right\} \text {. }
$$

As a self-adjoint operator on $L^{2}(X), \Delta_{X}$ has a spectral decomposition. This decomposition can be identified with the decomposition in the sense of (1.1). It is in this way that we obtain the Plancherel formula for $X$.

Theorem 4.2. The direct integral decomposition of $L^{2}(X)$ is as follows

$$
L^{2}(X) \cong \sum_{\varepsilon} \frac{1}{2 \pi i} \int_{i \mathbb{R}_{+}} L_{\varepsilon, \nu}^{2}(\Xi)|c(\varepsilon, \nu)|^{-2} d \nu+\sum_{\varepsilon, \nu} L_{\varepsilon, \nu}^{2}(\Xi)^{0}
$$

where the first sum is over $\varepsilon=0,1 ; c(\varepsilon, \nu)=2^{2 \rho+1} \pi^{\rho}|\Gamma(\nu) / \Gamma(\nu+\rho)|$ if $G=G_{2(2)}$ and $c(\varepsilon, \nu)=2^{2 \rho+1} \pi^{\rho}|\Gamma(\nu) / \Gamma(\nu+\rho)| \times\left|\tan \left(\frac{1}{2} \pi(\rho+\nu+\varepsilon)\right)\right|$ if 
$G=\operatorname{Spin}_{e}(3,4) ;$ the second sum is over $\varepsilon=0,1$ and $\nu>0$ for which $\nu+\rho$ is a positive integer and $\varepsilon \cong \rho+\nu-q(\bmod 2) ; L_{\varepsilon, \nu}^{2}(\Xi)^{0}$ is the unitary irreducible quotient of $L_{\varepsilon, \nu}^{2}(\Xi)$.

The theorem follows from Theorem 3.3 and the main results of [R] (Theorem 10, 12 and 13). The irreducible representations of $G$ occurring in the first sum in (4.1) are called the continuous series for $X$ and those occurring in the second sum are called the discrete series for $X$. As a consequence of the theorem, we have following two corollaries.

For $\nu \in i \mathbb{R}$ and $f \in D(X)$ we define $\hat{f}_{\varepsilon, \nu}(\xi) \in L_{\varepsilon, \nu}^{2}(\Xi)$ by

$$
\hat{f}_{\varepsilon, \nu}(\xi)=\int_{X}|(x, \xi)|^{-\rho+\nu} \operatorname{sign}^{\varepsilon}(x, \xi) f(x) d x .
$$

Corollary 4.3. Every $K$-finite functions $f \in D(X)$ can be written as

$$
\begin{aligned}
f(x)= & \sum_{\varepsilon} \frac{1}{2 \pi i} \int_{i \mathbb{R}_{+}} \int_{Y}|(x, y)|^{-\rho-\nu} \operatorname{sign}^{\varepsilon}(y) \hat{f}_{\varepsilon, \nu}(y) d y\left|2^{-\rho} c(\varepsilon, \nu)\right|^{-2} d \nu \\
& +\sum_{\substack{\varepsilon \equiv \rho+\nu_{0}-q \\
\rho+\nu_{0} \in \mathrm{N}, \nu>0}} \operatorname{res}{ }_{\nu=\nu_{0}}\left\{c(\varepsilon, \nu)^{-1} \int_{Y}|(x, y)|^{-\rho-\nu} \operatorname{sign}^{\varepsilon}(x, y) \hat{f}_{\varepsilon, \nu}(y) d y\right\} .
\end{aligned}
$$

Corollary 4.5. A polar semisimple homogeneous space has discrete series if and only if it has a compact Cartan subspace.

This corollary gives a proof of Theorem 2.5.

Remark 4.6. For the case $G / H=\operatorname{Spin}_{e}(3,4) / G_{2(2)}$ if we only sum over $\varepsilon=0$ in (4.1), we get the Plancherel formula for $G / H=\mathrm{SO}_{e}(3,4) / G_{2(2)}$. Note that the corresponding representations of $\operatorname{Spin}_{e}(3,4)$ factor through $\mathrm{SO}_{e}(3,4)$.

\section{Eigenspace representations on $X$.}

In this section we will discuss the two spaces of eigenfunctions of the Laplacian $\Delta_{X}$ under the regular representation of $G$. One is a subspace of smooth functions on $X$,

$$
C_{\varepsilon, \lambda}^{\infty}(X)=\left\{f \in C^{\infty}(X) \mid \Delta_{X} f=\lambda f, \text { and } f(-x)=(-1)^{\varepsilon} f(x)\right\} .
$$

The other is a subspace of $L^{2}$-functions on $X$,

$$
L_{\varepsilon, \lambda}^{2}(X)=\left\{f \in L^{2}(X) \mid \Delta_{X} f=\lambda f \text { and } f(-x)=(-1)^{\varepsilon} f(x)\right\} .
$$

We will also give a Poisson integral-type for $K$-finite eigenfunctions of $\Delta_{X}$. 
We set

$$
C_{\varepsilon, \nu}^{\infty}(\Xi)=\left\{\left.\phi \in C^{\infty}(\Xi)|\phi(\alpha \xi)=| \alpha\right|^{-\rho+\nu} \operatorname{sign}^{\varepsilon}(\alpha) \phi(\xi)\right\} .
$$

Restrict this space of functions to $Y$ is a linear isomorphism $C_{\varepsilon, \nu}^{\infty}(\Xi) \rightarrow$ $C_{\varepsilon}^{\infty}(Y)=\left\{\phi \in C^{\infty}(Y) \mid \phi(-y)=(-1)^{\varepsilon} \phi(y)\right\}$.

We define a map

$$
F_{\varepsilon, \nu}: C_{\varepsilon, \nu}^{\infty}(\Xi) \rightarrow C^{\infty}(X)
$$

by

$$
F_{\varepsilon, \nu} \phi(x)=\int_{Y}|(x, y)|^{-\rho-\nu} \operatorname{sign}^{\varepsilon}(x, y) \phi(y) d y .
$$

The integral converges for $\operatorname{Re}(\rho+\nu)<1$ and admits an interpretation by analytic continuation in $\nu$, as long as $\nu$ is not a positive integer congruence to $\varepsilon+1(\bmod 2)$.

Lemma 5.1 ([R] Lemma 5).

(i) The map $F_{\varepsilon, \nu}$ depends meromorphically on $\nu$ with simple poles for $\rho+\nu \in \mathbb{N}$ and $\equiv \varepsilon+1 \bmod 2$.

(ii) $F_{\varepsilon, \nu}$ is a G-map: $F_{\varepsilon, \nu} \pi_{\varepsilon, \nu}(g) \phi=R(g) F_{\varepsilon, \nu} \phi$, for any $\phi \in C_{\varepsilon, \nu}^{\infty}(\Xi)$.

(iii) For any $\phi \in C_{\varepsilon, \nu}^{\infty}(\Xi), \Delta_{x} F_{\varepsilon, \nu} \phi=\left(-\rho^{2}+\nu^{2}\right) F_{\varepsilon, \nu} \phi$.

Here $R$ denotes the regular representation.

\section{Proposition 5.2.}

(i) Every $K$-finite distribution $f$ on $X$ satisfying

$$
\Delta_{X} f=\lambda f
$$

for some $\lambda \in \mathbb{C}$ is an analytic function.

(ii) Fix a $K$-type $\tau$ and a complex number $\lambda$. The space of all $K$-finite analytic functions $f$ of type $\tau$ satisfying

$$
\Delta_{X} f=\lambda f
$$

has dimension equal to $\operatorname{dim} V_{\tau}$, which is independent of eigenvalue $\lambda$.

Proof. The statement (i) of the proposition follows from (a) of Lemma 3 in $[\mathbf{R}]$. The same lemma guarantee the dimension in (ii) is less than or equal to $\operatorname{dim} V_{\tau}$. Applying an argument of analytic continuation such as in Proposition 2.21 in $[\mathrm{OS}]$ one can get the other half inequality.

The following theorem and Theorem 5.5 below can be regarded as a Poisson integral formula for $X$. 
Theorem 5.3 (cf. [R, Theorem 6]). If $\rho+\nu$ is not an integer, then every $K$-finite distribution $f$ on $X$ satisfying

$$
\Delta_{X} f=\left(-\rho^{2}+\nu^{2}\right) f
$$

is an analytic function and can be represented in the form

$$
f(x)=\sum_{\varepsilon} \int_{Y}|(x, y)|^{-\rho-\nu} \operatorname{sign}^{\varepsilon}(x, y) \phi_{\varepsilon}(y) d y
$$

for unique $K$-finite function $\phi_{\varepsilon} \in C_{\varepsilon}^{\infty}(Y)$.

Corollary 5.4.

(i) If $\lambda=-\rho^{2}+\nu^{2}($ or $\rho+\nu)$ is not an integer (note that $\lambda$ is an integer if and only if $\rho+\nu$ is an integer), then $C_{\varepsilon, \lambda}^{\infty}(X)$ is irreducible under the regular representation of $G$. The map $F_{\varepsilon, \lambda}$ is an intertwining operator between $C_{\varepsilon, \pm \nu}^{\infty}(\Xi)$ and $C_{\varepsilon, \lambda}^{\infty}(X)$, and the $K$-finite part of $C_{\varepsilon, \pm \nu}^{\infty}(\Xi)$ and $C_{\varepsilon, \lambda}^{\infty}(X)$ are equivalent $(\mathfrak{g}, K)$-modules.

(ii) If $\lambda=\lambda_{0}=-\rho^{2}+\nu_{0}^{2}$ and $\rho+\nu_{0} \in \mathbb{N}, \equiv \varepsilon+q \bmod 2, \nu_{0}>0$, then the kernel of $\operatorname{res}_{\nu=\nu_{0}}\left\{c(\varepsilon, \nu)^{-1} F_{\varepsilon, \nu}\right\}$ is the $G$-stable subspace of $C_{\varepsilon, \nu_{0}}^{\infty}(\Xi)$ described by (cf. Theorem 3.2)

$$
-\left(m+\frac{1}{2}(p-2)\right)+\left(n+\frac{1}{2}(q+2)\right)<\nu_{0} .
$$

As representations of $G, C_{\varepsilon, \nu_{0}}^{\infty}(\Xi)$ and $C_{\varepsilon, \lambda_{0}}^{\infty}(X)$ have the same distribution character.

Now we turn our attention to the square-integrable eigenfunctions of $\Delta_{X}$. The following theorem is a consequence of Theorem 12 of $[\mathbf{R}]$ and Theorem 3.2 .

Theorem 5.5. For $\lambda=-\rho^{2}+\nu^{2}$, the eigenspaces $L_{\varepsilon, \lambda}^{2}(X)$ of $\Delta_{X}$ are irreducible under the regular representation of $G$. For $\rho+\nu=\rho+\nu_{0} \in \mathbb{N}, \equiv$ $\varepsilon+q(\bmod 2), \nu_{0}>0, \operatorname{res}_{\nu=\nu_{0}}\left\{c(\varepsilon, \nu)^{-1} F_{\varepsilon, \nu}\right\}$ induces an intertwining operator between an irreducible quotient $L_{\varepsilon, \nu}^{2}(\Xi)^{0}$ and $L_{\varepsilon, \lambda}^{2}(X)$. The corresponding $K$-finite functions in $L_{\varepsilon, \lambda}^{2}(X)$ are precisely the functions $f$ of the form

$$
f(x)=\operatorname{res}_{\nu=\nu_{0}}\left\{c(\varepsilon, \nu)^{-1} \int_{Y}(x, y)^{-\rho-\nu} \operatorname{sign}^{\varepsilon}(x, y) \phi(y) d y\right\}
$$

where $\phi$ is a $K$-finite function on $Y$.

\section{References}

[D] J. Dadok, Polar coordinate induced by actions of compact Lie groups, Trans. Amer. Math. Soc., 288(1) (1985), 125-137. 
[FJ] M. Flensted-Jensen, Discrete series for semisimple symmetric spaces, Annal of Math., 111 (1980), 253-311.

[HC] Harish-Chandra, Discrete series for semisimple Lie groups, I, II, Acta Math., 113 (1965), 241-318; 116 (1966), 1-111.

[HPTT] E. Heintze, R. Palais, C.-L. Terng and G. Thorbergsson, Hyperpolar actions and $k$-flat homogeneous spaces, J. reine angew. Math., 454 (1994), 163-179.

[H] J.S. Huang, Harmonic analysis on compact polar homogeneous spaces, to appear in Pacific Journal of Mathematics.

[K] T. Kobayashi, Discrete decomposition of the restriction of $A_{\mathfrak{q}}(\lambda)$ with respect to reductive subgroups and its applications, Invent. Math., 117 (1994), 181-205.

[OM] T. Oshima and T. Matsuki, A description of discrete series for semisimple symmetric spaces, Group Representations and Systems of Differential Equations, Advanced Studies in Pure Mathematics, 4 (1984), 331-390.

[OS] T. Oshima and S. Sekiguchi, Eigenspaces of Invariant Differential Operators on an Affine Symmetric Space, Inv. Math., 57 (1980), 1-81.

[PT] R. Palais and C.-L. Terng, A general theory of canonical forms, Trans. of Amer. Math. Soc., 300 (1987), 771-789.

[R] W. Rossmann, Analysis on real hyperbolic spaces, J. Funct. Anal., 30 (1978), 448477.

[W] N. Wallach, Real Reductive Groups, Volume I, II, Academic Press 1988, 1992.

Received December 15, 1994. The author was supported in part by NSF Grant DMS9306138 and RGC Competitive Earmarked Research Grant HKUST 588/94P.

The Hong Kong Univeristy of Science and Technology

Clear Water Bay, Kowloon

HONG KONG

E-mail address: mahuang@uxmail.ust.hk 


\title{
PACIFIC JOURNAL OF MATHEMATICS
}

\author{
Founded in 1951 by
}

\author{
$\begin{array}{ll}\text { E. F. Beckenbach (1906-1982) } & \text { F. Wolf (1904-1989) }\end{array}$
}

\section{EDITORS}

Sun-Yung A. Chang (Managing Editor) Robert Finn University of California

Los Angeles, CA 90095-1555

pacific@math.ucla.edu

\section{F. Michael Christ}

University of California

Los Angeles, CA 90095-1555

christ@math.ucla.edu

Nicholas Ercolani

University of Arizona

Tucson, AZ 85721

ercolani@math.arizona.edu
Stanford University

Stanford, CA 94305

finn@gauss.stanford.edu

Steven Kerckhoff

Stanford University

Stanford, CA 94305

spk@gauss.stanford.edu

Martin Scharlemann

University of California

Santa Barbara, CA 93106

mgscharl@math.ucsb.edu

\section{Gang Tian}

Massachusettes Institute of Technology

Cambridge, MA 02139

tian@math.mit.edu

\section{S. Varadarajan University of California Los Angeles, CA 90095-1555 vsv@math.ucla.edu \\ Dan Voiculescu \\ University of California \\ Berkeley, CA 94720 \\ dvv@math.berkeley.edu}

\section{SUPPORTING INSTITUTIONS}

ACADEMIA SINICA, TAIPEI

CALIF. INST. OF TECHNOLOGY

CHINESE UNIV. OF HONG KONG

HONG KONG UNIV. OF SCI. \& TECH.

KEIO UNIVERSITY

MACQUARIE UNIVERSITY

MATH. SCI. RESEARCH INSTITUTE

NEW MEXICO STATE UNIV.

OREGON STATE UNIV.

PEKING UNIVERSITY

RITSUMEIKAN UNIVERSITY

STANFORD UNIVERSITY

\author{
TOKYO INSTITUTE OF TECHNOLOGY \\ UNIVERSIDAD DE LOS ANDES \\ UNIV. OF ARIZONA \\ UNIV. OF BRITISH COLUMBIA \\ UNIV. OF CALIF., BERKELEY \\ UNIV. OF CALIF., DAVIS \\ UNIV. OF CALIF., IRVINE \\ UNIV. OF CALIF., LOS ANGELES \\ UNIV. OF CALIF., RIVERSIDE \\ UNIV. OF CALIF., SAN DIEGO \\ UNIV. OF CALIF., SANTA BARBARA
}

UNIV. OF CALIF., SANTA CRUZ

UNIV. OF HAWAII

UNIV. OF MELBOURNE

UNIV. OF MONTANA

UNIV. NACIONAL AUTONOMA DE MEXICO

UNIV. OF NEVADA, RENO

UNIV. OF OREGON

UNIV. OF SOUTHERN CALIFORNIA

UNIV. OF UTAH

UNIV. OF WASHINGTON

WASHINGTON STATE UNIVERSITY

The supporting Institutions listed above contribute to the cost of publication of this Journal, but they are not owners or publishers and have no responsibility for its contents or policies.

Manuscripts must be prepared in accordance with the instructions provided on the inside back cover.

The table of contents and the abstracts of the papers in the current issue, as well as other information about the Pacific Journal of Mathematics, may be found on the Internet at http://www.math.uci.edu/pjm.html.

The Pacific Journal of Mathematics (ISSN 0030-8730) is published monthly except for July and August. Regular subscription rate: $\$ 245.00$ a year (10 issues). Special rate: $\$ 123.00$ a year to individual members of supporting institutions.

Subscriptions, back issues published within the last three years and changes of subscribers address should be sent to Pacific Journal of Mathematics, P.O. Box 4163, Berkeley, CA 94704-0163, U.S.A. Prior back issues are obtainable from Kraus Periodicals Co., Route 100, Millwood, NY 10546.

The Pacific Journal of Mathematics at the University of California, c/o Department of Mathematics, 981 Evans Hall, Berkeley, CA 94720 (ISSN 0030-8730) is published monthly except for July and August. Second-class postage paid at Berkeley, CA 94704, and additional mailing offices. POSTMASTER: send address changes to Pacific Journal of Mathematics, P.O. Box 6143, Berkeley, CA 94704-0163.

\section{PUBLISHED BY PACIFIC JOURNAL OF MATHEMATICS at University of California,} Berkeley, CA 94720, A NON-PROFIT CORPORATION

This publication was typeset using AMS-LATEX,

the American Mathematical Society's TEX macro system.

Copyright (C) 1995 by Pacific Journal of Mathematics 


\section{PACIFIC JOURNAL OF MATHEMATICS}

\section{Volume $176 \quad$ No. $2 \quad$ December 1996}

One remark on polynomials in two variables

ENRIQUe ARTAl BARTOLO and PIERRETTE CASSOU-NOGUÈS

Divergence of the normalization for real Lagrangian surfaces near complex tangents XIANGHONG GONG

Classification of the stable homotopy types of stunted lens spaces for an odd prime JESUS GONZALEZ

Plancherel formulae for non-symmetric polar homogeneous spaces

JING-SONG HUANG

A uniqueness theorem for the minimal surface equation

JENN-FANG HWANG

Differential Galois groups of confluent generalized hypergeometric equations: an approach

using Stokes multipliers

Claudine Mitschi

Oscillatory theorem and pendent liquid drops

KIMIAKI NARUKAWA and TAKASHI SUZUKI

Local and global plurisubharmonic defining functions

ALAN NOELL

Specializations and a local homeomorphism theorem for real Riemann surfaces of rings

M. J. DE LA PUENTE

Eigenvalue comparisons in graph theory

GREGORY T. QUENELL

Applications of loop groups and standard modules to Jacobians and theta functions of isospectral curves

WILLI SCHWARZ

Bridged extremal distance and maximal capacity

ROBERT E. THURMAN

Imbedding and multiplier theorems for discrete Littlewood-Paley spaces

IGOR E. VERBITSKY

On constrained extrema

THOMAS VOGEL

Heat flow of equivariant harmonic maps from $\mathbb{B}^{3}$ into $\mathbb{C P} \mathbb{P}^{2}$

YUANLONG XIN

Proof of Longuerre's theorem and its extensions by the method of polar coordinates

ZHIHONG YU

Correction to: "Special generating sets of purely inseparable extension fields of unbounded exponent"

BONIFACE IHEMOTUONYE EKE 